\title{
DIE BYZANTINISCHE FASSUNG DES SPÄTANTIKEN BRIEFSTELLERS: ÜBERLIEFERUNG UND TEXTGESCHICHTE
}

\section{Dmitri A. Chernoglazov}

St. Petersburg State University, 7-9, Universitetskaya nab., St. Petersburg, 199034, Russian Federation; d_chernoglazov@mail.ru

"Epistolary styles" of Pseudo-Libanius (abbr. PL1), an ancient treatise on letter writing of the $5^{\text {th }}$ century, includes a theoretical introduction and sample letters. The examples refer to 41 types, as a rule, one example per type. Whereas the treatise itself, which forms the basis of ancient epistolary theory, has been studied well enough, its Byzantine and post-Byzantine versions have not been studied at all. In this article, one of these versions is analyzed - a collection of sample letters compiled on the basis of Pseudo-Libanius (abbr. PL2). Our research includes the following aspects: 1) Content and style of PL2. The names of the types, represented in PL2, and the texts of the samples, go back, mainly, to the treatise of Pseudo-Libanius. The method of reworking the source is analyzed: the text is expanded by rhetorical means - epithets, comparisons, proverbs and citations are regularly introduced. 2) The PL2 manuscript tradition. Mss. PL2, as a rule, coincides with the treatise of Pseudo-Libanius and enters into various combinations with it. Depending on the structure of the text, PL2-mss. are divided into 5 classes. The characteristic of each manuscript is given: not only of the treatise itself, but also its context in mss. is taken into account. 3) The PL2 text history. An attempt is made to reconstruct the archetype of PL2: it is demonstrated that initially it was not an appendix to "Epistolary Styles", but an independent collection of sample letters. Its supposed date is the $10^{\text {th }}$ century. 4) The purpose and function of PL2. It is assumed that PL2 was written in order to update the ancient sample letters. In mss. PL2 often acts as a transitional link between the simplest examples of Pseudo-Libanius and authentic letters of Byzantine authors. Perhaps the treatise was also used as a school textbook. In the Appendix all sample letters included in PL2, with their names and incipit, are listed. The critical edition of "Epistolary Styles" and all their Byzantine versions is being now prepared by the author of this article.

Keywords: Epistolary theory, sample letters, manuscript tradition, textual criticism, Ancient epistolography, Byzantine epistolography, Pseudo-Libanius, Characteres epistolici.

Die antike Brieftheorie ist im Detail untersucht und wohlbekannt. Ihre Fortsetzung und Entwicklung in Byzanz bleibt jedoch fast völlig unerforscht. Der Gegenstand des vorliegenden Artikels ist ein byzantinischer Briefsteller auf der Grundlage des spätantiken theoretischen Traktats des Pseudo-Libanios.

Der Briefsteller von Pseudo-Libanios / Pseudo-Proklos (weiter: PL1, edd.: Weichert

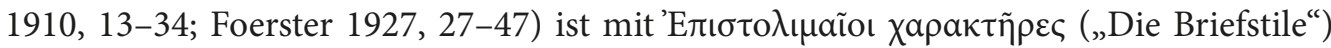

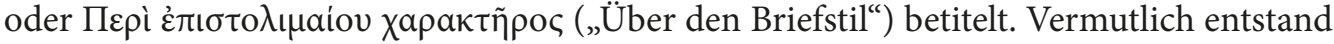

(C) St. Petersburg State University, 2017 
der Traktat im 5. Jh. In verschiedenen Hss. wird er regelmäßig entweder dem „Sophisten Libanios“, oder dem „Platoniker Proklos“ zugeschrieben. Die Forscher stimmen jedoch darin überein, dass beide Zuschreibungen falsch sind und der Name des echten Autors unbekannt ist (Koskenniemi 1956, 56-57; Zilliacus 1949, 48-51; Grünbart 2005,

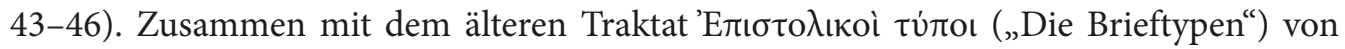
Pseudo-Demetrios (Weichert 1910, 1-12) bildet PL1 die Basis der spätantiken und frühbyzantinischen Brieftheorie.

PL1 enthält die folgenden Bestandteile:

1. Theoretische Einführung und Definition des Briefs (Weichert 19101, 13. 1-14. 9).

2. Eine Liste der 41 Brieftypen, die nach Inhalt und Ziel zu unterschieden sind (14. 10-15.4).

3. Definitionen von Brieftypen (15. 5-19.6)

4. Ratschläge über den Briefstil im Allgemeinen (19.7-21. 15)

5. Kurze Musterbriefe, regelmäßig ein Muster für jeden Typ (21. 16-34. 5)

PL1 ist in zwei Hauptversionen überliefert, die eine unterschiedliche Struktur haben. In der ersten, die nach dem Autornamen die „Libanios-Version“ genannt ist (Foerster 1927, 3-10), sind die Bestandteile in der oben erwähnten Reihenfolge eingeordnet; in der zweiten jedoch, die als „Proklos-Version“ gilt (Foerster 1927, 10-17), sind die Musterbriefe mit den entsprechenden Definitionen kombiniert. Welche dieser Versionen dem PL1Archetyp nähersteht, bleibt noch eine offene Frage. ${ }^{2}$

PL1 ist weder für die klassischen Philologen noch für die Byzantinisten terra incognita. Der kleine Traktat wurde zwar vielfach herausgegeben (Foerster 1927, 2126), in moderne Sprachen übersetzt (Malherbe 1988, 68-81; Malosse 2004, 21-39) und ausführlich studiert, doch immer nur im Kontext der spätantiken Epistolografie. Seine Bedeutung und Autorität während des ganzen byzantinischen Jahrtausends und sogar noch später, bis zum 19. Jh., ist bis heute nicht anerkannt (Chernoglazov 2017). PL1 wurde viele Jahrhunderte lang immer wieder kopiert, mit Zusätzen und Kommentaren versehen. Für unsere Forschung ist besonders wichtig, dass so neue Texte geschaffen wurden, die sich vom Archetyp wesentlich unterscheiden, diesen inhaltlich sogar um das Drei- bis Vierfache übertreffen, weshalb man bei ihnen eigentlich kaum noch von Versionen, als vielmehr von selbstständigen Traktaten sprechen kann, die auf PL1 Gründen. Einer dieser Traktate wird uns nachfolgend interessieren.

Der Briefsteller, den wir zu untersuchen beabsichtigen (im Folgenden PL2), ist eine Sammlung von Musterbriefen, die am häufigsten als Supplement zu PL1 überliefert sind. PL2 liegt bisher nur in einer Edition von V. Weichert vor (Weichert 1910, 37-66), die aber kaum ausreicht, da dafür nicht alle Hss. berücksichtigt wurden und einige Musterbriefe fehlen. Bis heute gibt es keine Forschungen zu diesem Text. Wann und wie entstand die Sammlung? Zu welchem Zweck und für welchen Leserkreis wurde sie geschrieben? Wie wurde sie genutzt? Auf diese Fragen zu antworten wäre sehr wichtig, um zu verstehen, wie

${ }^{1}$ Die Edition von R. Foerster und E. Richtsteig (Foerster 1927) ist zwar die neuere und bessere, doch werden wir auf die Edition von V. Weichert (Weichert 1910) verweisen, da PL1 nur dort zusammen mit dem uns interessierenden Traktat (s. unten) ediert ist und es uns das Zitieren und Vergleichen beider Versionen erleichtert, wenn die Paragrafen einheitlich nummeriert sind.

${ }^{2}$ J.Sykutris behauptet, dass die Proklos-Version die ursprüngliche sei. Seine Argumente erscheinen uns jedoch sehr problematisch (Sykutris 1928/1929). 
sich die antike Brieftheorie auf byzantinischem Boden entwickelte und wie sie im neuen System der mittelalterlichen Literatur funktionierte.

In diesem Artikel beschreiben wir zuerst den Inhalt und Stil von PL2 und konzentrieren uns dann auf dessen handschriftliche Tradition sowie auf die Textgeschichte. Den Abschluss werden einige Vermutungen über den Zweck und die Funktion von PL2 bilden.

\section{Inhalt und Stil}

Insgesamt umfasst PL2 64 Musterbriefe, die jedoch in keiner Hs. vollständig vorliegen. Der größte Zahl an Mustern ist in zwei Hss. überliefert: $V$ und Par (zu diesen Hss. s. unten). Die meisten Briefe sind durch ihre Titel bestimmten Typen zugeordnet, insgesamt 48. Diese stammen größtenteils aus PL1: Zu den 41 PL1-Typen wurden der „enkomiastische Brief“, der „Beratungsbrief“, der „Abratungsbrief“, der „wechselseitige Brief“ der „Versprechensbrief“ und zwei „Begrüßungsbriefe“ („an einen Freund“ und „an einen Geistlichen“) hinzugefügt. Jedem Typ sind zwischen einem und vier Muster zugeordnet. Einige Muster sind jedoch ohne Titel überliefert und mit keinem Typ verbunden. Im Anhang finden sich sämtliche PL2-Musterbriefe in alphabetischer Reihe der Titel und den initia. Ihre laufenden Nummer sind gemäß der Edition von V. Weichert angegeben. Wenn ein Typ aus PL1 entlehnt ist, wird der Titel fett ausgezeichnet und seine Nummer in PL1 in Klammern gesetzt.

Die meisten PL2-Musterbriefe hängen unmittelbar von entsprechenden Mustern in PL1 ab. So ist z. B. der „Mitleidsbrief“ in PL2 eine Überarbeitung seiner Entsprechung in PL1. Manche PL2-Muster, die zu keinem PL1-Typ gehören (epp. 61 $\gamma \delta, 63,86,87$ ), enthalten tatsächlich verschiedene Varianten des „Freundesbriefs“. Nur einige Texte sind von PL1 unabhängig, z. B.der „Unterrichtsbrief“ und der „Berichtsbrief“. Vier umfangreiche „Trostbriefe“ enthalten Parallelen untereinander, Es ist jedoch unmöglich, deren Verhältnis zum PL1-„Trostbrief“ zu bestimmen, da dieser verschollen ist.

Kennzeichen von PL2 ist die Methode, die der anonyme Autor benutzt, um seine Grundlage zu überarbeiten. Die Länge der Briefe wird durchweg deutlich ausgedehnt, jedoch in der Regel ohne Anreicherung konkreter Details. Rhetorische Mittel amplifizieren den Text: Metaphern, Vergleiche, Epitheta, Zitate und Sprichwörter. Außerdem werden die für die byzantinische Epistolografie typischen Freundschaftsmotive systematisch eingeführt, heidnische Gestalten entfallen, stattdessen wird die christliche Thematik regelmäßig entwickelt. Beispielhaft für diese Methode ist der „Klagebrief“; nachfolgend die Muster dieses Typs in PL1 und PL2 ${ }^{3}$ :

PL1, ep. 24

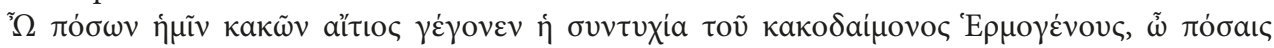

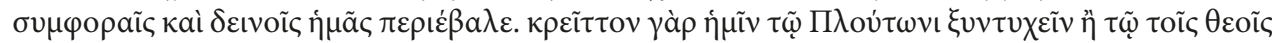
$\dot{\varepsilon} \chi \theta \rho \tilde{\omega}$.

„Wie viele schlechte Dinge hat uns das Treffen mit dem bösen Hermogenes verursacht! Mit wie vielen Unglücken und Gefahren hat es uns umgeben! Es ist wirklich besser für uns, uns eher mit Pluton zu treffen, als mit dem Feind der Götter!“

${ }^{3}$ Es sollte beachtetet werden, dass ein weiteres Muster des „Klagebriefes“ in PL2 erhalten ist (ep. 68 $\beta$ ), eine gekürzte Version des hier angeführten (ep. 68a). 
PL2, ep. 68a

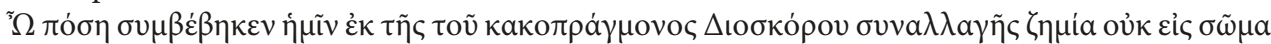

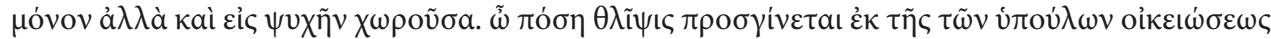

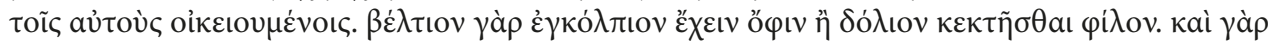

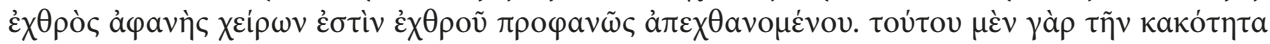

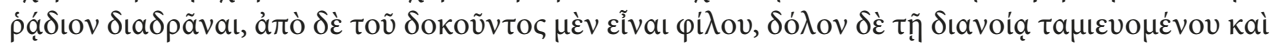

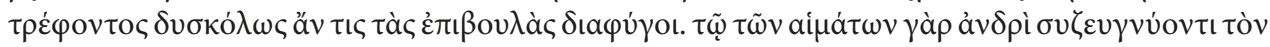

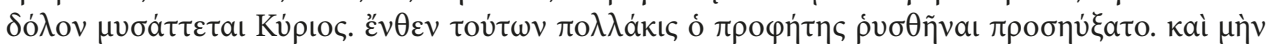

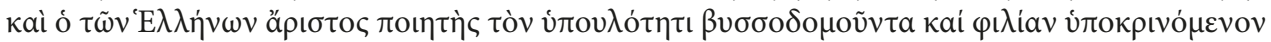

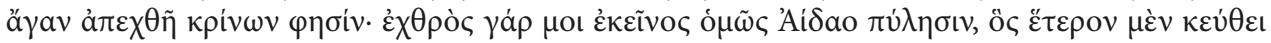

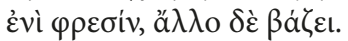

„Wie viel Schaden hat uns das Treffen mit dem Bösewicht Dioskoros verursacht, der nicht nur den Körper, sondern auch die Seele betrifft! Wie viel Bedrückung entsteht durch das Zutrauen zu den Heimtückischen, für diejenigen, die mit solchen Leuten Umgang haben. Es ist wirklich besser, eine Schlange im Busen zu haben, als einen falschen Freund zu bekommen. Denn ein unerkannter Feind ist schädlicher als ein Feind, der offen hasst. Es ist einfacher, die Bosheit des Letztgenannten zu vermeiden; wenn man jedoch ein Freund zu sein scheint, aber das Falsche in Gedanken verbirgt und pflegt, ist es schwierig, die Hinterlist zu vermeiden. Der Herr scheut den Blutgierigen und Falsches Ausführenden (cf. Ps 5:7). Deshalb bat der Prophet mehrfach [Gott], vor diesem gerettet zu werden (Ps 58:3; 138:19). So hielt auch Homer, der beste Dichter der Heiden, den Menschen, der die Freundschaft vorgibt, aber geheime Absichten hat, für sehr verhasst, indem er sagt (Hom. Il. 9: 312-313, übers. von J.H. Foss):

Denn mir verhasst ist jener, so sehr wie des Aïdes Pforten, wer ein Eines im Herzen verbirgt, und ein Anderes redet."

\section{Die handschriftliche Tradition}

Die handschriftliche Tradition von PL2 ist sehr komplex: die Querverbindungen zwischen PL1 und PL2 sind höchst vielfältig. Zudem weicht die Reihenfolge der Musterbriefe in den Hss. stark voneinander ab. Je nach der Textstruktur der Briefsammlung kann man alle PL2 enthaltenden Hss. in fünf Klassen unterteilen, deren Merkmale noch näher zu bestimmen sein werden. Für jede Hs. werden die folgenden Angaben aufgeführt: Datum; Folio-Angaben, auf denen PL1 und PL2 erhalten sind; PL2-Muster, die in der betreffenden Hs. vorliegen; unmittelbarer Kontext des Traktats in der Hs. (vorangehende und nachfolgende Texte sind erwähnt); allgemeine Information zum Inhalt der Hs., wobei hauptsächlich festzuhalten sein wird, wenn weitere unserem Traktat thematisch naheliegende Werke in der Hs. erhalten sind, z. B. Briefformulare, Briefsammlungen, theoretische Traktate und Lehrbücher zu Grammatik und Rhetorik; ausgewählte Sekundärliteratur.

Zur Klasse I, die am häufigsten vorkommt, sind die Hss. zu rechnen, in denen die PL2Muster eine gesonderte Gruppe bilden. Diese Gruppe ist entweder unabhängig überliefert oder als Anhang an PL1 angeschlossen. Die Reihenfolge ist in allen Hss annähernd die gleiche, unterscheidet sich aber von der Reihenfolge, die in allen Hss. von PL1 herrscht. Während z. B. der erste Typ und das erste Briefmuster in PL1 der "Belehrungsbrief“ ist, fängt die PL2-Sammlung der ersten Klasse mit dem „Lobesbrief“ an, der sich in PL1 an Platz 26 befindet. 
Vind = Vind. phil. gr. 342 (11. Jh.)

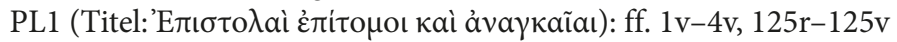

PL2 (ohne Titel): ff. 125v-134v.

Epp.: 60, 61 $\alpha \beta \gamma \delta, 62 \alpha \beta \gamma, 63-65,62 \delta, 66,67 \alpha, 68 \beta, 69-76$

Der Kontext ist schwer zu rekonstruieren, da die ursprüngliche Reihenfolge der Folia wesentlich korrumpiert ist.

Inhalt: Beide Versionen sind als Teile einer großen epistolografischen Sammelhandschrift überliefert, die auch Briefe von Johannes Chrysostomos und verschiedenen Autoren des 10. Jh. enthalten: Nikolaos I. Mystikos, Theodoros von Kyzikos, Bardas Monachos, Niketas Paphlagon, Niketas Magister und Leo von Synada.

Lit.: Hunger 1961, 435-437; Tziatzi-Papagianni 2012, 30*-36*. Die Hs. wurde von den Editoren von PL1 und PL2 nicht berücksichtigt.

$V=$ Vaticanus gr. 306 (13-14. Jh.)

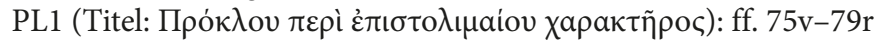

PL2 (ohne Titel und Intervall): ff. 79r-88v.

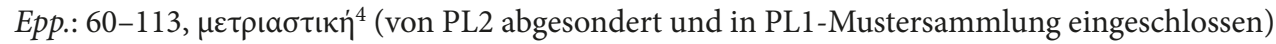
Kontext: Nikephoros Basilakes, Progymnasmata - Niketas Magister, Briefe; Prokopios von Gaza, Briefe

Lit.: Mercati, Cavalieri 1923, 452-453; Weichert 1910, LVI; Foerster 1927, 17-18. Variae lectiones sind in der Edition von V. Weichert berücksichtigt.

Heid = Palatinus Heidelbergensis gr. 356 (14. Jh.)

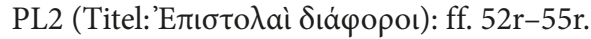

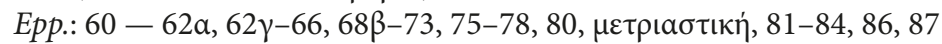

Kontext: Michael Psellos, Briefe — (ohne Zwischentitel) Symeon Magister, Briefe

Inhalt: Die Handschrift enthält auch viele andere antike und frühbyzantinische Briefe (Brutus, Libanios, Basileios der Große, Gregorios von Nazianz, Julianos u. a.)

Lit.: Stevenson 1885, 203-207; Weichert 1910, LXXIV; Foerster 1927, 10. Variae lectiones sind dennoch nicht berücksichtigt.

Marc = Marcianus gr. Z. 521 (14. Jh.)

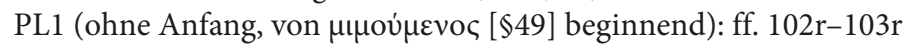

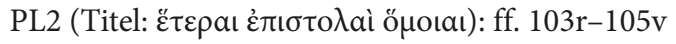

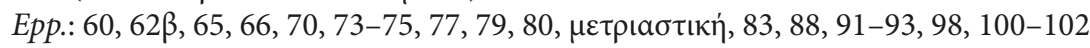

Kontext: Galenus, Definitiones medicae - Brutus, Briefe (vielleicht ist die Reihenfolge der Folia korrumpiert)

Inhalt: Der Briefsteller geht der Sammlung von antiken und frühbyzantinischen Briefen voraus.

Lit.: Weichert 1910, LVI; Foerster 1927, 18

Havn = Havniensis gr. 1985 (14-15. Jh.)

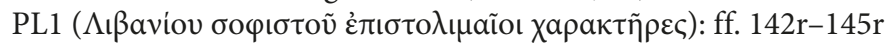

PL2 (ohne Überschrift): ff. 145r-155v.

Epp.: 60-86

Kontext: Chronologica varia - Michael Psellos, De omnifaria doctrina

Inhalt: Die Handschrift enthält auch viele antike und frühbyzantinische Briefe (Libanios, Basileios, Gregorios, Johannes Chrysostomos, Theodoros Studites)

Lit.: Schartau 1994, 205-213; Weichert 1910, LVI; Foerster 1927, 4. Variae lectiones sind in der Edition von V. Weichert berücksichtigt.

${ }^{4} \mathrm{Zu}$ diesem Muster s. Anhang 
Laur $=$ Laurentianus Plutei 59. 5 (15. Jh.)

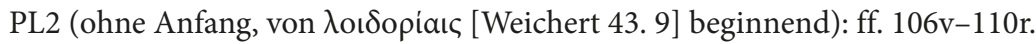

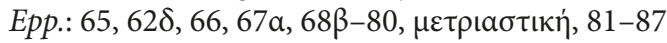

Kontext: Alkiphron, Briefe - Ende der Hs.

Inhalt: Der Briefsteller ist ans Ende der Sammelhandschrift antiker Briefe kopiert (Phalaris, Brutus, Aeschines u. a.)

Lit.: Bandini 1961, 491-493; Weichert 1910, LVI; Foerster 1927, 18-19. Variae lectiones sind in der Edition von V. Weichert berücksichtigt.

Vat = Vaticanus gr. 1733 (15. Jh.)

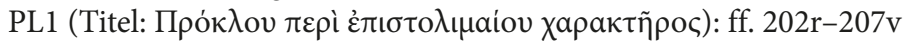

PL2 (ohne Titel und Intervall): ff. 207v-214r.

Epp.: 60-628, 76-78, 63-75, 79-84

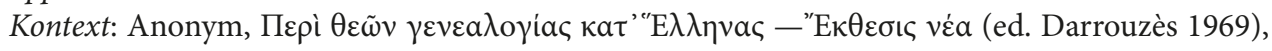
eine Sammlung von Brief- und Anredeformularen zu kirchlichen und weltlichen Würdenträgern. Inhalt: Die Handschrift enthält verschiedene theoretische Traktate über Rhetorik (Maximos Planudes, Herodianus u. a.), dazwischen auch die „Brieftypen“ von Pseudo-Demetrios; viel Lehrmaterial auf Vulgärgriechisch ( $\theta \varepsilon \mu \alpha \tau$ т $\rho а \varphi$ ía, Briefmuster)

Lit.: Giannelli 1961, 115-126; Weichert 1910, LVI; Foerster 1927, 18. Variae lectiones sind in der Edition von V. Weichert berücksichtigt.

Vatop = Vatopedinus 12 (15. Jh.)

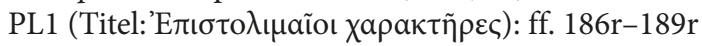

PL2 (ohne Titel und Intervall): ff. 189r-196v.

Epp.: 60-64, 66, 68-86

Kontext: Basileios der Große, ep. 2 - Nonnos von Panopolis (Pseudo-), In S. Gregorii Nazianzeni or. 39 historiae $1-24$

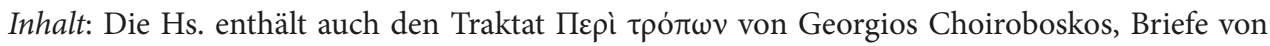
Johannes Chrysostomos und verschiedene Briefformulare

Lit.: Lamberz 2006, 72-87. Die Hs. ist von den Editoren von PL1 und PL2 nicht berücksichtigt.

In den Hss. von Klasse 2 bilden die PL2-Muster keine gesonderte Gruppe, sind aber voneinander getrennt und mit den entsprechenden PL1-Mustern kombiniert: Das PL2-Muster des „Bittbriefs“ (ep. 94) z. B. folgt dem PL1-Muster desselben Typs (ep. 3); zwei PL2-Muster des „aufhetzenden“ Briefes, die sich in den Hss. von Klasse 1 fern voneinander befinden (epp. 65, 108), bilden hier eine thematisch einheitliche Gruppe zusammen mit dem „aufhetzenden“ PL1-Brief (ep. 20); PL2-Muster sind in der Regel als

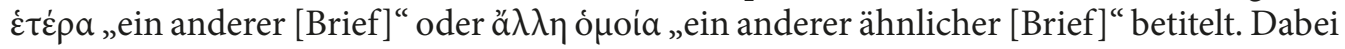
folgt der „Beratungsbrief“ (ep. 92) auf die „Belehrungsbriefe“ (epp. 90, 91), da der erste Brieftyp in PL1 als ähnlich dem letzten erwähnt ist; aus dem gleichen Grunde schließt der „enkomiastische Brief“ (ep. 71) an den „Lobesbrief“ (ep. 70) an. Der in den Hss. von Klasse 2 erhaltene PL2-Text weicht - manchmal wesentlich - vom Text der Klasse 1 ab. Unterschiede zwischen beiden Klassen sind in epp. 70-73 besonders leicht zu verfolgen: Es genügt, den apparatus criticus von V. Weichert zu betrachten.

Par $=$ Parisinus gr. 2671 (15. Jh.)

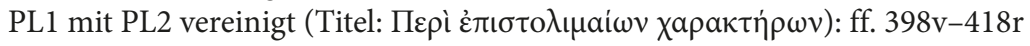




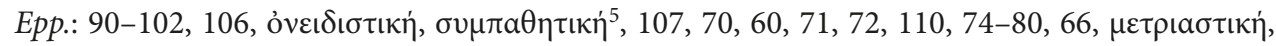
$82-84,103-105,65,108,67 \beta, 68 \alpha, 68 \beta, 62 \beta, 61 \alpha, 61 \beta, 69,64,81,85$; zwei Muster vom Trostbrief $(62 \gamma, 62 \delta)$ getrennt überliefert (s. unten)

Inhalt: Der Briefsteller gilt als Teil einer großen Sammlung, die theoretische Anweisungen und Briefmuster umfasst. Der Inhalt der Sammlung ist folgender:

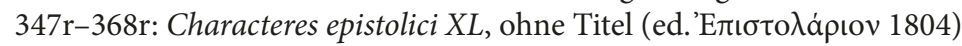

368v-387r: leere Blätter

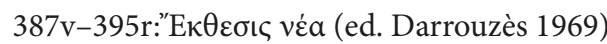

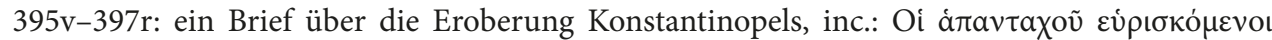

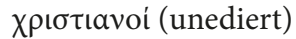

398r: die Liste der 40 Brieftypen als Tabelle dargestellt

398v-418r: PL1 mit PL2

418r: ein Abschnitt der rhetorischen Synopsis von Joseph Rhakendytes, das Briefschreiben be-

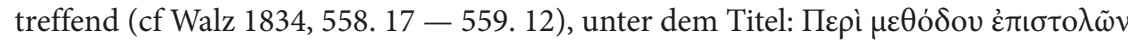

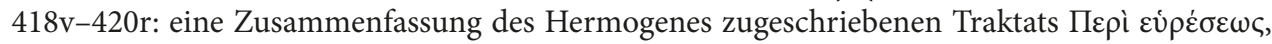

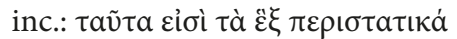

421r-421v: zwei Muster des „Trostbriefs“ aus PL2 (Weichert 62 $\gamma, 62 \delta$ )

422r-432v: zwei „Trostbriefe“ von Photios (Laourdas - Westerink 1983-1988, ep. 245, 234)

433r-433v: zwei Briefe von Demetrios Kydones (Loenertz 1956-1960, ep. 420, 122)

Lit.: Omont 3, 1888, 23-24; Darrouzès 1969, 7; Weichert 1910, LVIII-LXII; Foerster 1927, 16-17. Variae lectiones sind in der Edition von V. Weichert berücksichtigt.

Laurent $=$ Laurentianus Plutei 55. 7 (15. Jh.)

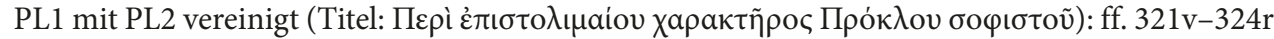

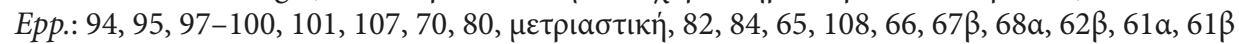

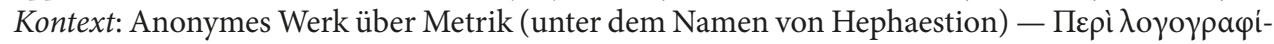

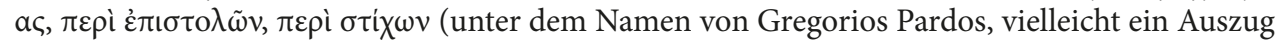
aus seinem Werk, s. Komines 1960, 56)

Inhalt: Die Handschrift enthält verschiedene grammatische Werke (z. B. Herodianus, die Eỉkóveৎ von Philostratos mit Epimerismen), eine rhetorische Theorie und Briefsammlungen von Brutus und Phalaris.

Lit.: Bandini 1961, 244-268; Weichert 1910, LVIII-LXII; Foerster 1927, 15. Variae lectiones sind in der Edition von V. Weichert berücksichtigt.

Bar = Baroccianus 125 (16. Jh.)

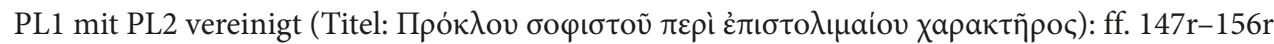

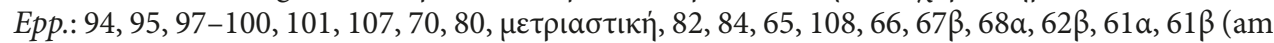
engsten verwandt dem Laur. Plut. 55. 7)

Inhalt: Der Briefsteller ist ein Teil der Briefsammlung folgenden Inhalts:

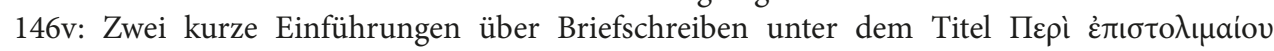

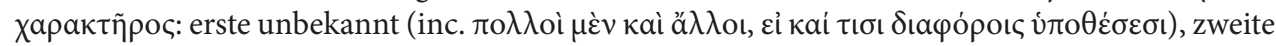
von Philostratos (Philostr. VS. II. 33. 3)

147r-156r: PL1 mit PL2

156v: kurze Bemerkungen, überwiegend grammatischen Inhalts.

$157 \mathrm{r}-199 \mathrm{v}$ : Briefsammlung von Synesios von Kyrene (86 Briefe)

200r-208v: 100 Briefformulare von Demetrios Chrysoloras

Lit.: Coxe 1969, 200-204; Weichert 1910, LVIII-LXII; Foerster 1927, 15-16. Variae lectiones sind in der Edition von V. Weichert berücksichtigt.

${ }^{5} \mathrm{Zu}$ diesen zwei Mustern s. Anhang 
Vatic $=$ Vaticanus gr. 875 (13. Jh.)

Einige PL2-Muster gekürzt, mit PL1-Mustern vereinigt und verschmolzen (Titel: $\chi \alpha \rho \alpha \kappa \tau \tilde{\eta} \rho \varepsilon \varsigma$

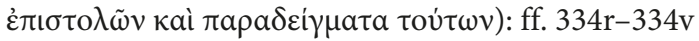

Eрp.: 90 (gekürzt), $2+75$ (gekürzt), $11+102$ (gekürzt und miteinander verschmolzen), $\sigma v \mu \pi \alpha \theta \eta \tau \iota \kappa \dot{~}+62 \delta$ (gekürzt und miteinander verschmolzen), $22+66$ (gekürzt und miteinander verschmolzen).

Kontext: Briefe von Theophylaktos Simokatta — verschiedene Epigramme

Inhalt: Die Hs. enthält auch das Lexikon von Pseudo-Zonaras und verschiedene grammatische Werke (Michael Psellos, Niketas von Serrai)

Lit.: Schreiner 1988, 24-30. Die Hs. ist von den Editoren von PL1 und PL2 nicht berücksichtigt.

Die Klasse 3 ist nur in einer Hs. vertreten. Die PL2-Briefe ergänzen nicht die PL1Muster, aber ersetzen sie. Der Text enthält also eine kurze theoretische Einleitung und 44 Muster zu 44 Brieftypen (41 PL1-Typen sowie der „Abratungsbrief“, der „,enkomiastische Brief“ und der „Beratungsbrief“). Alle Briefe sind PL2-Muster, die jedoch meistens der Reihenfolge von PL1 - i. e. mit dem „Belehrungsbrief“ beginnend - folgen und mit den Definitionen wie in der Proklos-Version von PL1 zusammengestellt sind. ${ }^{6}$ Der Text der Muster ist dem Text von Klasse 2 fast identisch, der - wie wir hingewiesen haben - von der Klasse 1 deutlich abweicht.

\begin{abstract}
Har $=$ Harleianus 5566 (14. Jh.)

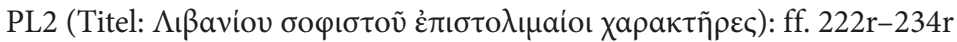

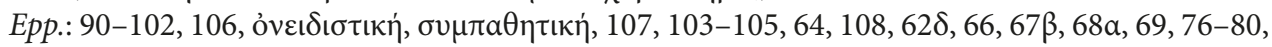

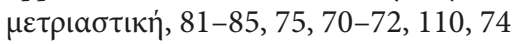

Kontext: Synesios von Kyrene, De dono astrolabi - Ders., De regno.

Inhalt: Die Handschrift enthält eine große Sammlung antiker und frühbyzantinischer Briefe: Alkiphron, Brutus, Phalaris und Synesios von Kyrene, sowie auch andere Werke von Synesios.

Lit.: Nares 1808, 277; Foerster 1927, 10. Variae lectiones sind von den Editoren nicht berücksichtigt.
\end{abstract}

Der Klasse 4 kann man die PL1-Hss. zurechnen. Nur ein oder zwei PL2-Muster sind darin eingedrungen. Diese Muster ergänzen entsprechende PL1-Muster, ersetzen sie oder füllen die Lakunen, wo das PL1-Muster im Verlauf der Textgeschichte verloren ging. Dies betrifft drei PL2-Muster: den „ermunternden Brief“ (ep. 77), einen der „Trostbriefe“ (ep. $62 \beta$ ) und den „Mischbrief“ (ep. 85).

Der „ermunternde Brief“"von PL2 (ep. 77) ist auf den Platz des „Trostbriefs“ (dessen PL1-Muster wohl schon damals verschollen war) in den folgenden Hss. gesetzt: Paris. gr. 2881; Vat. Ottob. gr. 339; Ambros. Q5. sup.; Laur. Plut. 60. 14; Berol. gr. 308; Paris. Suppl. gr. 165; Wolfenbüttel. 3132; Vat. gr. 82; Paris. gr. 1389; Palat. gr.358; Hierosol. Patr. Bibl. 217. Zu diesen Hss. und den in ihnen erhaltenen PL1-Versionen s. Foerster 1927, 5-7.

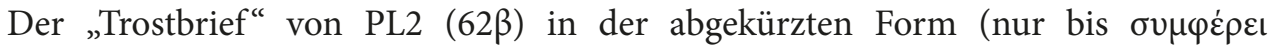
$\pi \alpha \rho \alpha \lambda \alpha \mu \beta \dot{\alpha} v o v \tau$ : Weichert 1910, 28. 20) ist in den folgenden Hss. ebenfalls auf den Platz des verschollenen PL1-„Trostbriefes“ gesetzt: Paris. gr. 1630; Paris. gr. 2551; Bodl. misc. 104; Vind. phil. 149; Heid. Pal. 43. S.: Foerster 1927, 13-15.

${ }^{6}$ Die Ausnahme besteht darin, dass die Muster von sechs Typen („Tadelbrief“, „Lobesbrief“, „enkomiastischer", „didaktischer", „entlarvender" und „,verrufender" Brief) ans Ende gesetzt sind, i. e. nach dem „Mischbrief“, der in der PL1-Reihenfolge der letzte ist. 
Der „Mischbrief“ von PL2 (ep. 85) ist vor dem PL1-Muster des Mischtyps (ep. 41) in den folgenden Hss. überliefert: Paris. gr. 2881; Vat. Ottob. gr. 339; Ambros. Q5. sup.; Laur. Plut. 60. 14. Derselbe PL2-„Mischbrief“ ersetzt das PL1-Muster (ep. 41) in den folgenden Hss.: Berol. gr. 308; Paris. Suppl. gr. 165; Wolfenbuttel. 3132; Vat. gr. 82; Paris. gr. 1389; Palat. gr.358; Hierosol. Patr. Bibl. 217. Man kann auch eine gekürzte PL1-Version in Bodl. Auct. T. 4 . 4 erwähnen: hier besteht der Traktat aus der Liste der Brieftypen, ihren Definitionen und nur einem Muster - dem „Mischbrief“ von PL2 (ep. 85). Zu dieser Hs. s. Foerster 1927, 9.

Klasse 5 umfasst Handschriften, in denen die Briefmuster von PL2 ohne Titel und theoretische Einleitung überliefert, in bunte Briefsammlungen inkorporiert sowie mit anderen Briefen gemischt sind. Gelegentlich erscheinen sie mit den Mustern von PL1 kombiniert.

$V t=$ Vaticanus gr. 1753 (15. Jh.)

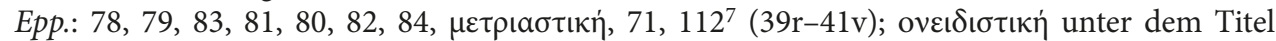

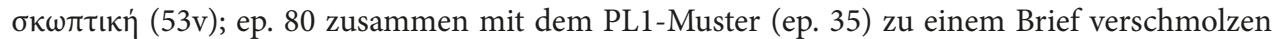
$(54 \mathrm{r}-54 \mathrm{v})$.

Inhalt: Die Sammlung, in der diese Muster erhalten sind, besteht aus 106 Briefen verschiedener Autoren (37r-60v), darunter: Libanios, Basilios, Photios und Theodoros Patrikios und Sakellarios. Die Briefe dieser und anderer, unbekannter Autoren sind miteinander vermischt. Im Titel vieler Briefe steht weder der Name des Autors noch der des Adressaten, wohl aber der Brieftyp $^{8}$ oder, noch konkreter, die Briefsituation sowie gelegentlich der soziale Status des Adressa-

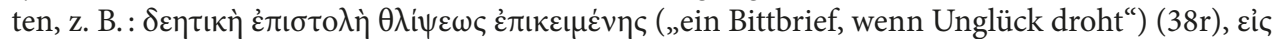

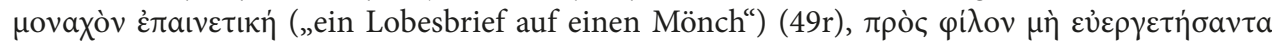

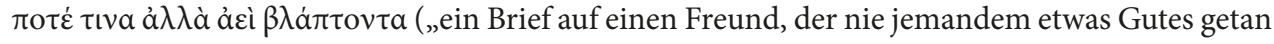
hat, diesen vielmehr immer schädigt") (55r) etc. Meist sind die Briefe nach Brieftyp und Briefsituation gruppiert: Vier „Lobes- und Dankbriefe“ stehen zusammen (49r-50v), des Weiteren drei

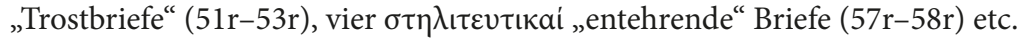

Lit.: Canart 1970, 36-47. Die Hs. ist von den Editoren von PL1 und PL2 nicht berücksichtigt.

Baroc $=$ Baroccianus 216 (15. Jh.)

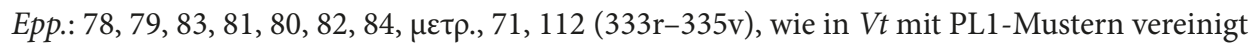
Inhalt: Die Handschrift enthält einen Teil der Sammlung, die uns aus Vt bekannt ist (331r-335v). Lit.: Coxe 1969, 376-383; Weichert 1910, LVIII-LX; Foerster 1927, 17. Variae lectiones sind von den Editoren nicht berücksichtigt.

$A c q=$ Laurentianus Acquisti e Doni 39 (16. Jh.)

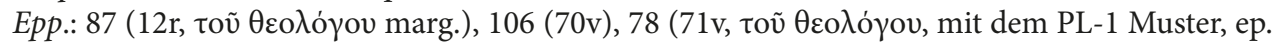
33 , vereinigt), $62 \beta-62 \gamma(103 \mathrm{r}-103 \mathrm{v})$

Die PL1- und PL2-Muster sind in eine große Briefsammlung (5r-137r) eingeschlossen, die auch Briefe von Basileios von Kaisareia, Johannes Chrysostomos, Theodoros Studites, Photios, Michael Psellos, Theophylaktos von Achrida und anderen, unbekannten Autoren enthalten (insgesamt 63 Briefe). Es gibt zudem drei Musterbriefe aus einer späteren Version von Pseudo-Libanios

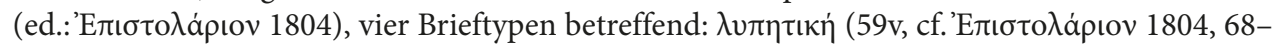

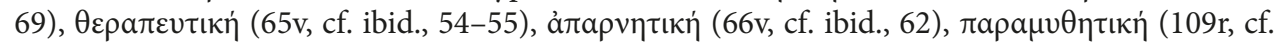

7 Epp. 78, 79, 83, 81, 80, 82, 84 sind mit den PL1-Muster vereinigt

${ }^{8}$ Nicht alle Typ-Bezeichnungen sind aus dem PL1, PL2 oder dem Traktat „Brieftypen“ von PseudoDemetrios entnommen. 
ibid., 57-58), mit anderen Briefen gemischt. Wie in $V t$ sind die Briefe nicht nach den Autoren, sondern nach den Brieftypen gruppiert. So ist z. B. eine Reihe von Trostbriefen und Trostreden

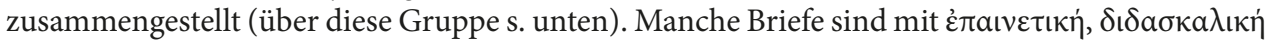

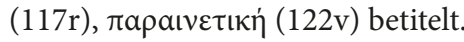

Lit.: Rostagno, Festa 1893, 197-199. Die Hs. ist von den Editoren von PL1 und PL2 nicht berücksichtigt.

Plut = Laurentianus Plutei 11. 13 (14. Jh.)

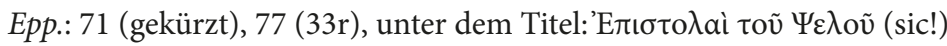

Kontext: Philo, De vita Moysis liber primus - Lucianus, verschiedene Werke bis zum Ende der Hs.

Lit.: Bandini I 509-510. Die Hs. ist von den Editoren von PL1 und PL2 nicht berücksichtigt.

Parisinus gr. 2022 (14. Jh.)

Ep. $62 \gamma(176 \mathrm{v}-177 \mathrm{r})$

Kontext: Der Brief (ohne Titel) ist in die Briefsammlung des Gregorios Kyprios eingeschlossen.

Inhalt: Die Hs. enthält eine Sammlung von frühbyzantinischen und spätbyzantinischen Briefen: Libanius, Gregorios Nazianzenos, Prokopios von Gaza, Gregorios Kyprios u. a.

Lit.: Omont 2, 1888, 180; Lameere 1937, 69-70. Die Hs. ist von den Editoren von PL1 und PL2 nicht berücksichtigt.

\section{Die Textgeschichte}

Wir haben alle uns bekannte Hss., in denen PL2-Musterbriefe erhalten sind, aufgelistet, beschrieben und versuchsweise in Klassen eingeteilt. Wie wir schon a priori bemerkt haben, ist die handschriftliche Tradition dieser Sammlung sehr instabil. Es lassen sich jedoch Vermutungen über den Archetyp der Sammlung und die Textgeschichte des Traktats im Allgemeinen anstellen.

Bezüglich der Struktur vom PL2-Archetyp kann man Folgendes vermuten:

Zum einen waren in ihm, wie in den Hss. der Klasse 1, PL2-Muster zusammengestellt und nicht mit den entsprechenden PL1-Mustern kombiniert. Es ist sehr wahrscheinlich, dass die PL2-Briefe, die ursprünglich in einer gesonderten Sammlung vorkamen, später in die Struktur des antiken Traktats PL1 inkorporiert und nach dessen Kategorien verteilt wurden. Ein gegenteiliger Prozess ist nur schwer vorstellbar. So, scheint die Form der Klasse 1 dem Archetyp am nächsten zu stehen.

Zum zweiten war PL2 ursprünglich kein Supplement zu PL1, sondern eine selbstständige Mustersammlung mit eigenem Titel, genau in der Form, in der der Traktat in einer der ältesten Hss., der Heid, überliefert ist. Es ergibt sich daraus, dass PL2 in seinen zahlreichen Hss. in Kombination mit sehr unterschiedlichen Versionen von PL1 vorkommt. In Havn etwa ist er mit der ursprünglichen Libanios-Version verbunden; in $V$ und Vat schließt er an eine andere Variante derselben Version an; in Par, Laurent und Bar ist er in die Proklos-Version integriert; in der ältesten Hs. Vind wurde er mit einer gänzlich unbekannten, gekürzten PL1-Version verbunden, die vielleicht mit dem in Paris. gr. 1760 erhaltenen Text (273r-275r) verwandt ist.

Wie kann der Archetyp von PL2 datiert werden? Einerseits wurde PL1 ungefähr im 5. Jh. geschrieben - das ist ein terminus post quem für PL2; andererseits entstand die älteste PL2-Hs. Vind im 11. Jh. - das ist ein terminus ante quem. Eine genauere Datierung des Traktats wäre eine schwierige Aufgabe, da die Briefe historischer Realien oder 
zeitbedingter sprachlicher Besonderheiten fast vollständig entbehren. Die Anredeformen und andere Elemente des Briefzeremoniells entsprechen völlig den Etiketteregeln der frühund mittelbyzantinischen Periode. Ein konkretes historisches Detail ist nur in einem PL2Brief vorhanden (ep. 113; Weichert 1910, 66. 13): Der Autor bittet den Adressaten, ihm zu

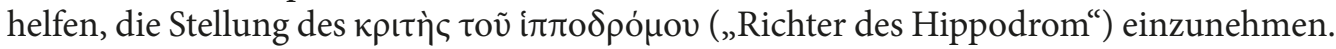
Dieses Amt ist nur vom 10. Jh. bekannt (Oikonomidès 1972, 323). Diese Datierung lässt sich nicht auf den ganzen Traktat übertragen: Dieser eine Brief ist nur in einer einzigen Hs., dem Vat, überliefert und befindet sich dort außerdem am Ende der Sammlung, sodass er auch ein spätere Zusatz sein kann. Es gibt aber noch ein anderes Argument, das uns veranlasst, PL2 dem 9.-10. Jh. zuzurechnen: In drei ältesten Hss. (Vind, V, Heid) befindet sich PL2 in unmittelbarer Umgebung von Briefen des 10.-11. Jh. von Niketas Magistros, Symeon Logothetes, Michael Psellos u. a. Besonders kennzeichnend ist Heid, wo die Briefe von Symeon Logothetes unseren Traktat ohne irgendeinem Zwischentitel oder Intervall fortsetzen und einen einheitlichen Text mit ihm bilden. Die gemischten Briefsammlungen $V t$ und $A c q$ enthalten viele Briefe vom 9.-10. Jh. Es muss betont werden, dass eine solche Nachbarschaft für die nur in PL1 enthaltene Hss. durchaus nicht typisch ist. Nur zwei Ausnahmen sind uns bis jetzt bekannt: Cod. Ambr. B4. sup., in dem die Briefe von Photios erhalten sind; Cod. Bodl. Auct. T.4. 4. mit den Briefen von Niketas Magistros, Symeon Logothetes und anderen Schriftstellern des 10. Jh.; aber sogar in diesen zwei Hss. sind PL1 und die mittelbyzantinischen Briefe sehr weit voneinander entfernt. In anderen Hss. schließt PL1 zwar häufig an verschiedene Briefsammlungen an, doch sind diese Briefe normalerweise antik und frühbyzantinisch, seltener aus palaiologischer Zeit. PL2 hingegen ist in Hss. eng mit mittelbyzantinischen Briefen verbunden. Eine solche Regelmäßigkeit lässt vermuten, dass PL2 sich zusammen mit diesen Briesammlungen während der makedonischen Zeit herausbildete.

Wie wir schon gezeigt haben, wurde der PL2-Archetyp mit verschiedenen PL1Versionen kontaminiert und trat mit diesen in vielfältige Verbindungen. Am häufigsten wurde die Sammlung einfach als Supplement dem antiken Briefsteller angefügt, wobei die Reihenfolge des Archetyps meistens unverändert blieb; dabei jedoch wurden viele Muster ausgelassen. So entstanden die Hss. der Klasse 1. In anderen Fällen benutzte man eine systematischere Methode: Die PL2-Muster wurden in die Struktur von PL1 (in der ProklosVersion) eingefügt und mit entsprechenden PL-1 Briefen kombiniert. Manchmal, wie in Vatic, wurden sie auch mt PL-1 Mustern verschmolzen. So entstanden die Hss. der Klasse 2. Einerseits sah das Ganze so stärker geregelt ${ }^{9}$ aus, andererseits jedoch verschwanden manche Muster, die zu keinem PL1-Typ gehörten, z. B.beide „Begrüßungsbriefe“ oder der „Versprechensbrief“. Die Fortsetzung dieses Prozesses bestand darin, dass PL2-Briefe, die mit PL1-Muster kombiniert wurden, diese zu verdrängen begannen, da sie vielleicht aktueller und nützlicher erschienen, woraus die Klasse 3 entstand. Während der mittelund spätbyzantinischen Periode war PL2 sehr verbreitet, weshalb viele PL1-Hss., ohne gründlich überarbeitet zu werden, um einige PL2-Muster ergänzt. Besonders betraf es den „Trostbrief“, dessen ursprüngliches Muster verschollen war: So entstand die Klasse 4. Gleichzeitig wurde PL2 nicht immer als geschlossener Text aufgefasst; seine Fragmente nämlich, zwischen einem und zwölf Musterbriefen umfassend, wurden in gemischte Briefsammlungen eingebettet - so bildeten sich die Hss. der Klasse 5 aus. Wahrscheinlich

9 Ein besonderes Bestreben zur Regelmäßigkeit findet man beim Verfasser von Par, der auch die Definitionen von den kombinierten PL1- und PL2 Mustern getrennt und alphabetisch geordnet hat. 
geschah die Verteilung von PL2 meistens aus dem folgenden Grund: Ein PL2-Muster (entweder allein, oder mit einem PL1-Brief kombiniert) wurde aus dem Briefsteller herausgezogen und in eine Gruppe von echten oder fingierten Briefen eingeschlossen, die einem und demselben Brieftyp angehörten. So sind in $V t$,Vorwurfsbrief “ und „spottender Brief“ aus PL2 in eine thematisch einheitliche Gruppe, die sechs „Schmähbriefe" und „spottende Briefe“ umfasst, eingefügt worden (53v-54v); ähnlich finden sich in Acq zwei PL2-,Trostbriefe“ in eine umfangreiche Gruppe von Trostbriefen und Trostreden $(83 \mathrm{v}-117 \mathrm{r})$ eingeschlossen, die auch drei Trostbriefe von Theodoros Studites (Fatouros 1992, epp. 497, 498, 454), drei Trostbriefe von Theophylaktos von Achrida (Gautier 1986,

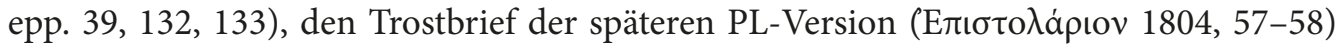
und andere Texte enthält. Vielleicht aus demselben Grund befinden sich zwei PL2Trostbriefe $(62 \gamma, 62 \delta)$ außerhalb des Briefstellers - sie sind mit zwei echten Trostbriefen von Photios zusammengestellt.

\section{Zweck und Funktion}

Abschließend könnte man Einiges darüber sagen, wofür PL2 verfasst wurde und wie er funktionierte. Als der ursprüngliche Traktat PL1 geschrieben wurde, sollte er kurzes Handbuch fürdie Briefkunst werden — und wirklich sind diedort vorliegenden Briefmuster den spätantiken Papyrusbriefen stilistisch ähnlich. In byzantinischer Zeit jedoch konnten diese knappen und schlichten exempla keineswegs als Objekte der Nachahmung gelten. Vielleicht deswegen wurde der Traktat PL2 geschrieben. Höchstwahrscheinlich geschah das in der makedonischen Renaissance, als die Byzantiner aufs Neue das Erbe der Antike entdeckten und Literaturgattungen des Altertums, darunter die Epistolografie, wieder belebten (Kazhdan 2006). So muss man es wohl als notwendig erachtet haben, die Brieftheorie zu erneuern und einen aktuellen Briefsteller zu schaffen.

Die neue Briefsammlung ergab sich tatsächlich aktuell für die byzantinischen Leser. Es sei daran erinnert, dass die meisten PL2-Muster Überarbeitungen von PL1-Mustern sind: Ursprünglich kurze Briefe wurden gerade um solche Elemente erweitert, die für die byzantinische Epistolographie sehr wichtig sind (Karlsson 1959): den rhetorischen Schmuck, das Thema der Freundschaft und christliche Motive. Im Ergebnis wurden neue Musterbriefe echten byzantinischen Briefen so ähnlich, dass sie - im Gegensatz zu den PL1-Mustern - eine Möglichkeit bekamen, sich mit echten Briefen zu mischen und selbst als Briefe verschiedener byzantinischen Autoren betrachtet zu werden. So schreibt man in Acq die PL2-Muster dem „Theologen“, i. e. Gregorios Nazianzenos zu; in Paris findet sich ein PL2-Muster in die Briefsammlung von Gregorios Kyprios eingeschoben, sodass man ihn als einen echten Brief des Byzantiners aus dem 13. Jh. deuten könnte (Lameere 1937, 69-70), wenn es in keiner anderen Hss. überliefert wäre.

PL2 konnte, mit PL1 auf unterschiedliche Weisen kombiniert, eine mittlere Stufe zwischen Theorie und Praxis sein: PL2-Musterbriefe konnten den Übergang erleichtern von den einfachsten, alten Mustern zu den echten Briefen eines Synesios von Kyrene oder Gregorios Nazianzenos. Diese Funktion wird von seiner handschriftlichen Tradition bezeugt: PL2 ist fast in allen Hss. mit Briefsammlungen verschiedener Autoren zusammengesetzt. Nicht selten ( $V$, Heid, Marc, Par, Bar) befindet sich der Briefsteller am Anfang eines solchen $\dot{\varepsilon} \pi ı \tau \tau o \lambda a ́ p ı v v$, manchmal auch mit einer zusätzlichen theoretischen Einleitung versehen (Par, Bar). So ergibt sich ein einheitliches Handbuch, das aus diesen 
Teilen besteht: theoretische Einführung, einfachste Muster (PL1), kompliziertere, der Praxis nahestehende Muster (PL2) und Briefe antiker oder byzantinischer Autoren. Das Einzige, das der Leser (der Schüler?) in diesem Handbuch nicht fand, waren Informationen über Anredeformen zu Vertretern verschiedener sozialer Stufen und andere formalen Aspekten eines Briefs. Deshalb wurden PL1 und PL2 in manchen Hss. auch mit einem Briefsteller anderen Typs zusammen kopiert, der nur Anrede- und Schlussformeln enthält:

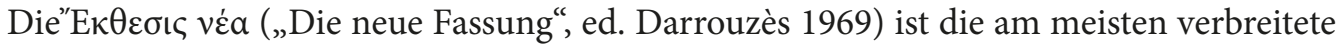
Version dieses Briefstellers, der in zwei Hss. (Par, Vat) mit PL2 gekoppelt ist.

Wir haben PL1 und PL2 Handbücher für Briefkunst genannt. Könnten sie auch Schulbücher in Byzanz gewesen sein? Wenn ja, auf welcher Stufe des Bildungsprozesses wurden sie benutzt? Derzeitig ist es schwierig, diese Frage zu beantworten: Das Lehrprogramm der byzantinischen Schulen ist in keiner byzantinischen Quelle systematisch dargelegt (Constantinides 1982). Man kann nur drei Dinge festzustellen: Zum einen wurden PL1 und PL2 häufig zusammen mit den theoretischen Traktaten kopiert, die man in der Schule als Handbücher für Grammatik und Rhetorik benutzte, z. B. mit den Werken von Dionysios Thrax, Herodianos, Georgios Choiroboskos, Maximos Planudes u. a. (Hunger 1978, 10-18) Ein typisches Beispiel ist Vat, in der PL1 und PL2 unmittelbar nach den „Progymnasmata" von Nikephoros Basilakes überliefert sind. Zweitens wurde die Briefkunst mit hoher Wahrscheinlichkeit in der byzantinischen Mittelschule gelernt: Davon überzeugt uns das Kompendium von Gregorios Pardos (von dem ein Auszug im Übrigen mit PL2 in

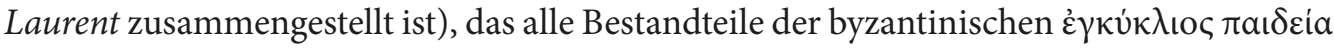
umfasst und darunter auch ein Kapitel über die Briefkunst enthält (Komines 1960). Weitere theoretische Handbücher byzantinischer Zeit für Epistolographie liegen uns nicht vor, abgesehen von verschiedenen Versionen des Pseudo-Libanios sowie kürzere, in Byzanz weniger verbreitete „Brieftypen“ von Pseudo-Demetrios. Schließlich kann man bestä-

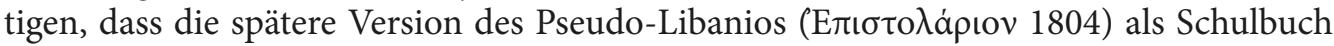
der Rhetorik benutzt wurde, da sein Text fast in allen Hss. mit zahlreichen rhetorischen Scholien überliefert ist. Vielleicht verdrängte dieser Traktat, der uns in vielen Hss. vom 14.-19. Jh. bekannt ist, frühere Handbücher und an erster Stelle PL2 (mit PL1 vereinigt), dem es im Laufe der Jahrhunderte nicht gelang, eine beständige Form anzunehmen.

Das ist der Stand unserer derzeitigen Beobachtungen. Sobald die kritische Edition aller Versionen von Pseudo-Libanios vorliegt, die jetzt von uns vorbereitet wird, werden wir diese Ergebnisse überprüfen und um Details erweitern können.

Anhang

\begin{tabular}{|c|c|c|}
\hline № & Titel & Incipit \\
\hline 81 & 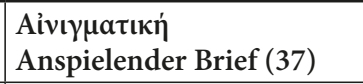 & 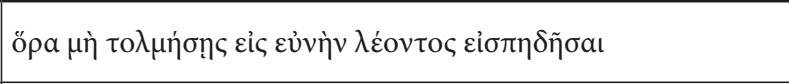 \\
\hline 87 & 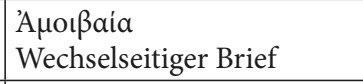 & 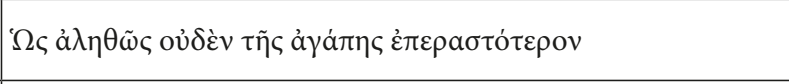 \\
\hline 78 & 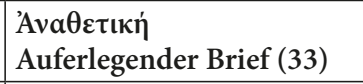 & 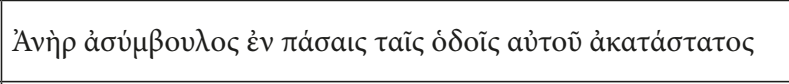 \\
\hline 105 & 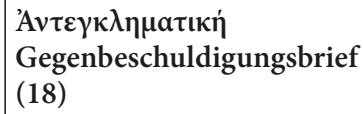 & 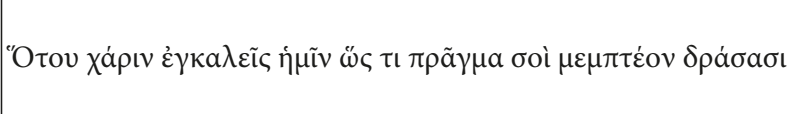 \\
\hline
\end{tabular}




\begin{tabular}{|c|c|c|}
\hline № & Titel & Incipit \\
\hline 64 & 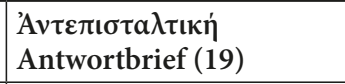 & 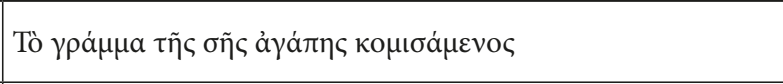 \\
\hline $67 a$ & \multirow{2}{*}{ 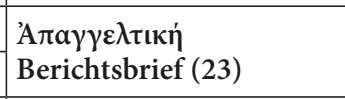 } & 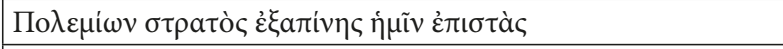 \\
\hline $67 \beta$ & & 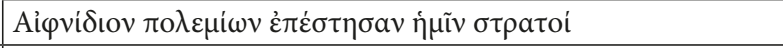 \\
\hline 101 & 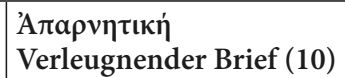 & 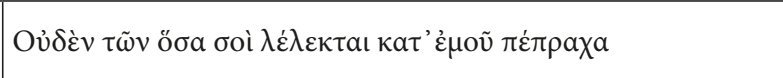 \\
\hline 100 & 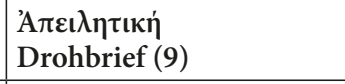 & 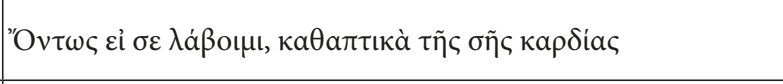 \\
\hline 79 & 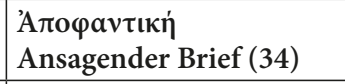 & 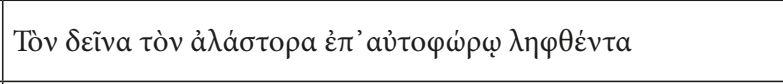 \\
\hline 74 & \begin{tabular}{|l|}
$\Delta \iota \alpha \lambda \eta \tau \iota \kappa \eta ́$ \\
Verrufender Brief (29)
\end{tabular} & 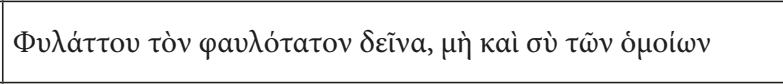 \\
\hline 72 & 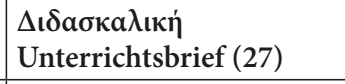 & 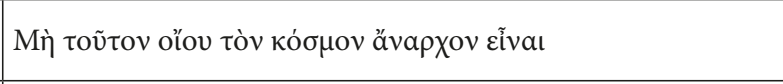 \\
\hline 71 & 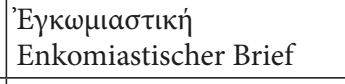 & 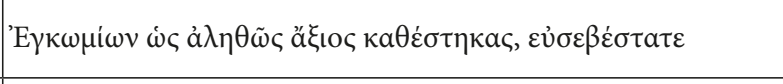 \\
\hline 96 & 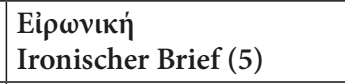 & 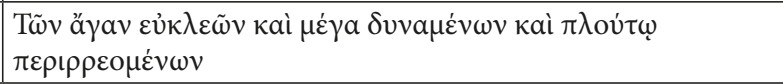 \\
\hline 73 & \multirow{2}{*}{$\begin{array}{l}' E \lambda \varepsilon \gamma \kappa \tau \iota \kappa \eta ' ~ \\
\text { Entlarvender Brief (28) }\end{array}$} & 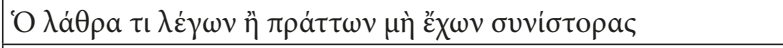 \\
\hline 110 & & 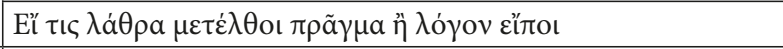 \\
\hline 88 & 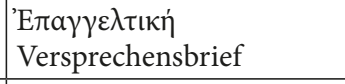 & 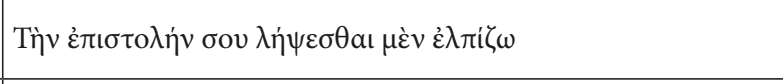 \\
\hline 60 & \multirow{2}{*}{ 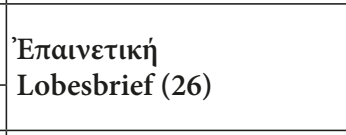 } & 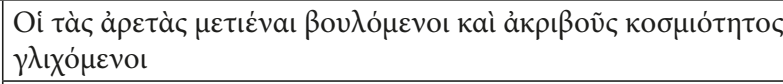 \\
\hline 70 & & 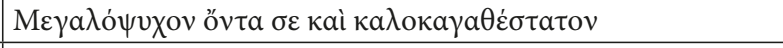 \\
\hline 75 & 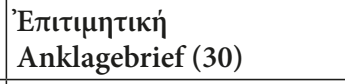 & 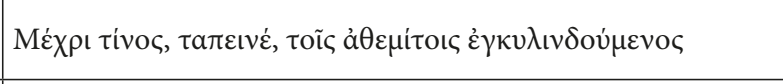 \\
\hline 76 & 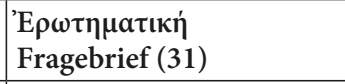 & 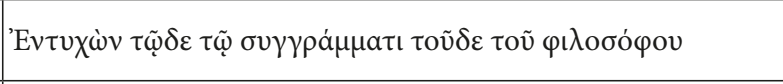 \\
\hline 84 & 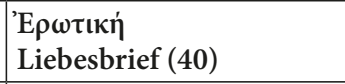 & 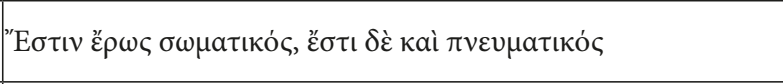 \\
\hline 99 & 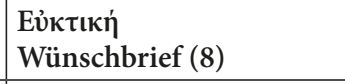 & 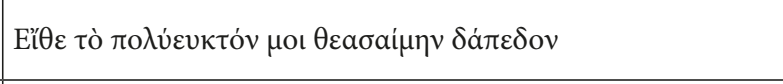 \\
\hline 97 & 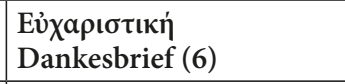 & 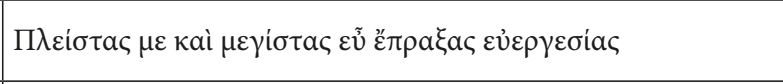 \\
\hline 107 & 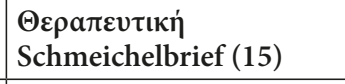 & 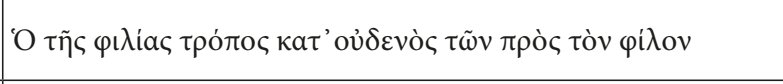 \\
\hline $83=111$ & 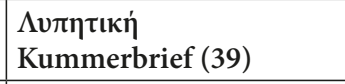 & 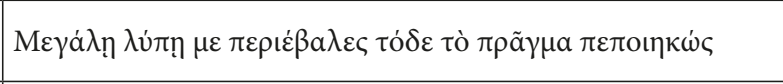 \\
\hline 93 & 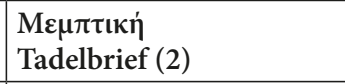 & 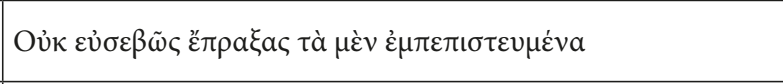 \\
\hline 106 & 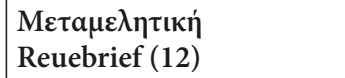 & 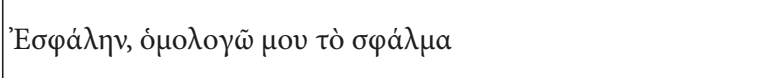 \\
\hline- & 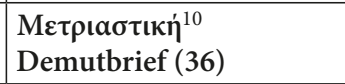 & 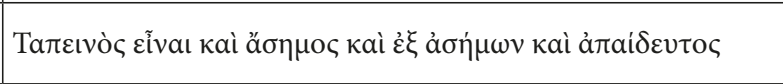 \\
\hline
\end{tabular}

${ }^{10}$ Der Brief ist nur teilweise ediert (Weichert 1910, 51) 


\begin{tabular}{|c|c|c|}
\hline № & Titel & Incipit \\
\hline 85 & 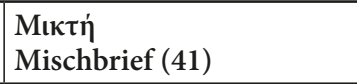 & 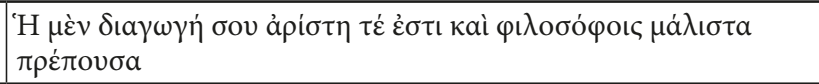 \\
\hline- & 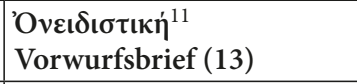 & 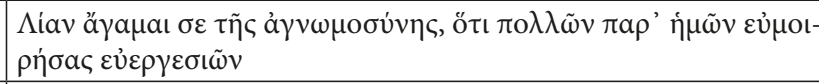 \\
\hline 102 & 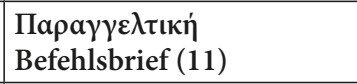 & 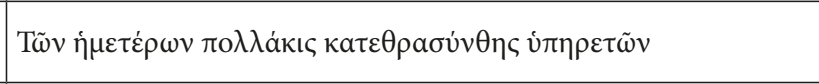 \\
\hline 77 & 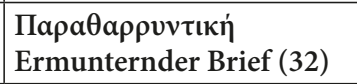 & 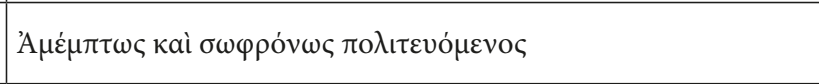 \\
\hline 90 & 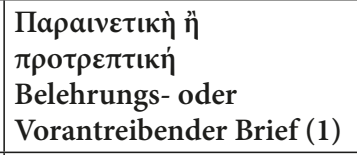 & 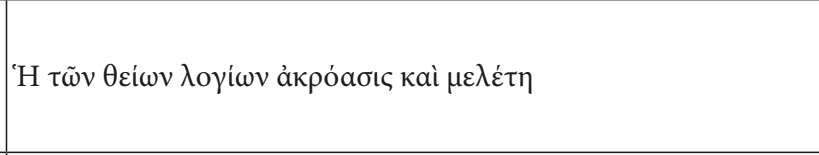 \\
\hline 91 & 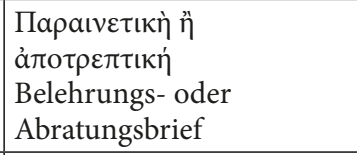 & 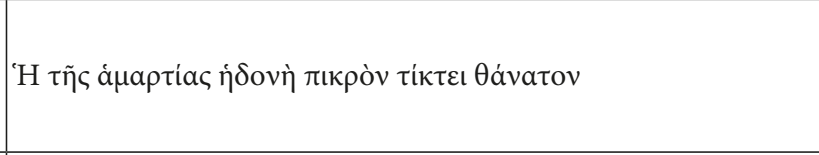 \\
\hline 94 & 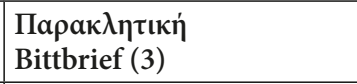 & 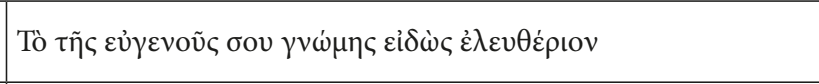 \\
\hline 104 & 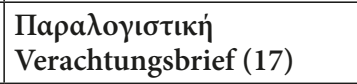 & 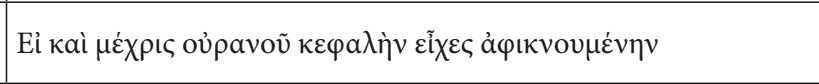 \\
\hline $62^{\alpha}$ & \multirow{4}{*}{ 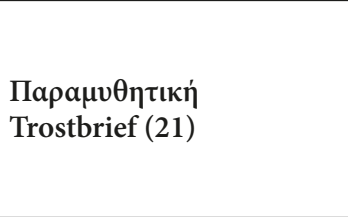 } & 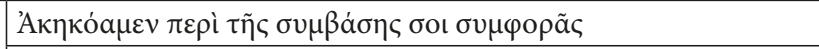 \\
\hline $62 \beta$ & & 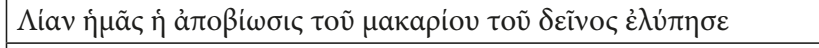 \\
\hline $62 \gamma$ & & 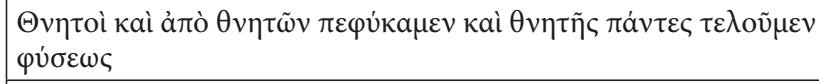 \\
\hline $62 \delta$ & & 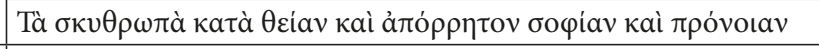 \\
\hline 65 & \multirow{2}{*}{ 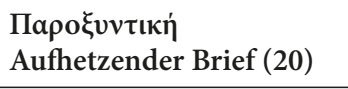 } & 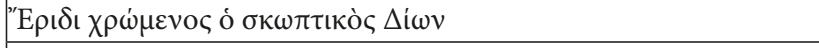 \\
\hline 108 & & 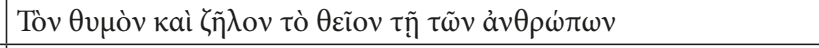 \\
\hline $61 \alpha$ & \multirow{2}{*}{ 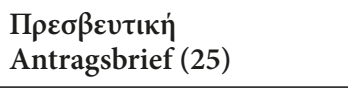 } & 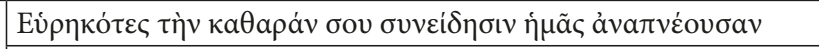 \\
\hline 69 & & 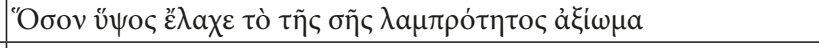 \\
\hline 63 & 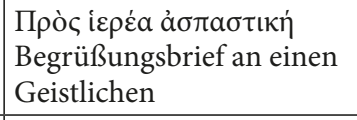 & 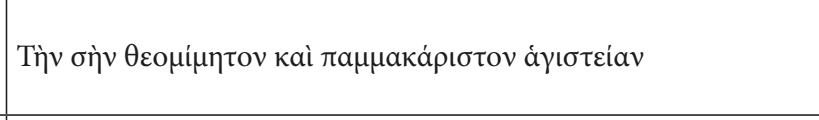 \\
\hline 86 & 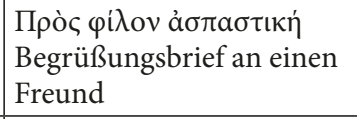 & 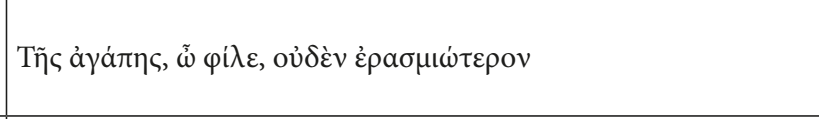 \\
\hline 80 & 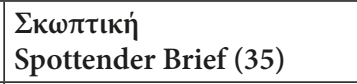 & 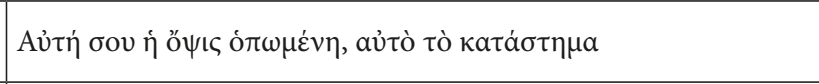 \\
\hline 103 & 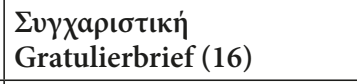 & 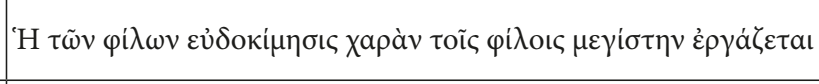 \\
\hline 92 & $\begin{array}{l}\Sigma v \mu \beta o v \lambda \varepsilon v \tau \iota k \eta ́ \\
\text { Beratungsbrief }\end{array}$ & 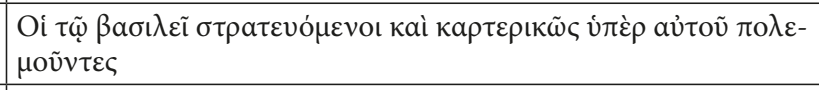 \\
\hline - & $\begin{array}{l}\Sigma v \mu \pi \alpha \theta \eta \tau \iota \eta^{12} \\
\text { Mitleidsbrief (14) }\end{array}$ & 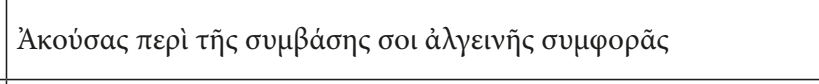 \\
\hline 95 & 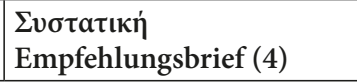 & 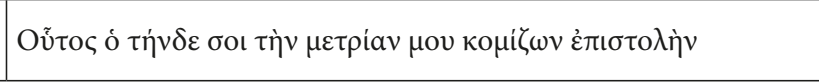 \\
\hline
\end{tabular}

${ }^{11}$ Der Brief ist unediert

12 Der Brief ist unediert 


\begin{tabular}{|c|c|c|}
\hline № & Titel & Incipit \\
\hline $68 a$ & \multirow{2}{*}{$\begin{array}{l}\Sigma \chi \varepsilon \tau \lambda \iota \alpha \sigma \tau \iota \kappa \eta ́ \\
\text { Klagebrief (24) }\end{array}$} & 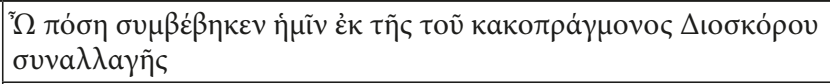 \\
\hline $68 \beta$ & & 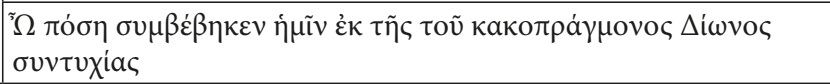 \\
\hline 66 & 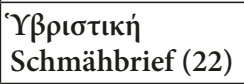 & 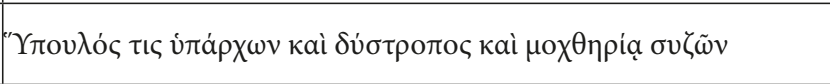 \\
\hline 82 & 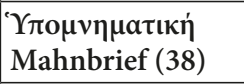 & 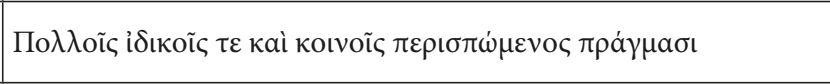 \\
\hline 98 & 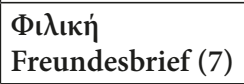 & 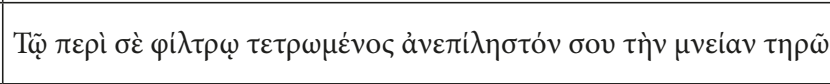 \\
\hline $61 \beta$ & - & 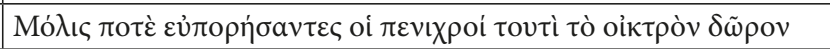 \\
\hline 109 & - & 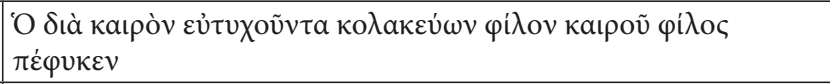 \\
\hline 89 & - & 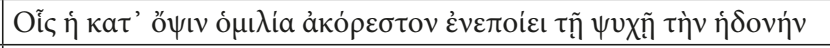 \\
\hline 113 & - & 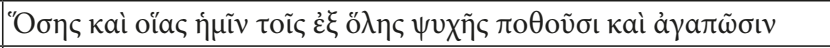 \\
\hline 112 & - & 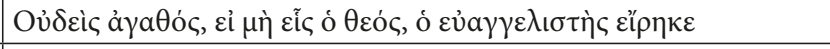 \\
\hline $61 \delta$ & - & 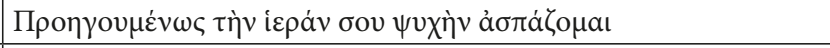 \\
\hline $61 \gamma$ & - & 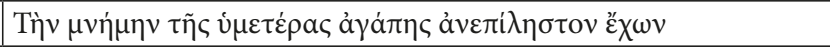 \\
\hline
\end{tabular}

\section{Literaturhinweise}

Bandini A. M. Catalogus codicum manuscriptorum Bibliothecae Medicae Laurentianae. Vol.1-2. Lipsiae, Zentral-Antiquariat der Deutschen Demokratischen Republik, 1961.

Canart P. Codices Vaticani graeci. Codices 1745-1962. Typis Polyglottis Vaticanis, 1970.

Chernoglazov D.A. „Ot Psevdo-Libanija do Nikodima Svjatogortsa: evoljutsija rannevizantijskogo pis' movnika" [Von Pseudo-Libanios bis Nikodemos Hagioreites: Entwicklung des frühbyzantinischen Briefstellers]. Izvestija Ural' skogo Federal' nogo Universiteta (Serija 2) 2017, 19, 68-82.

Constantinides C. N. Higher Education in Byzantium in the Thirteenth and Early Fourteenth Centuries: (1204 - ca. 1310). Nicosia, Cyprus Research Centre, 1982.

Coxe H. O. Bodleian Library. Quarto catalogues, I. Greek manuscripts. Oxford, Bodleian Library, 1969.

Darrouzès J. Ekthésis néa. Manuel des pittakia du XIVe siècle. Revue des Études Byzantines 1969, 27, 5-127.

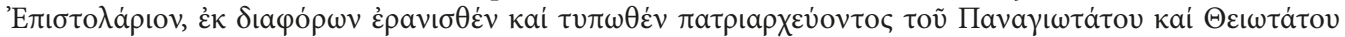

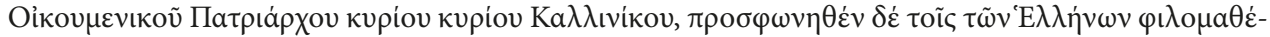

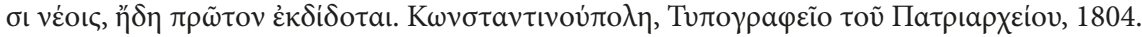

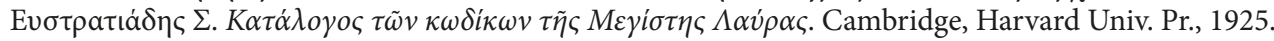

Fatouros G. (ed.). Theodori Studitae epistulae. Vol. 1-2. Berolini, de Gruyter, 1992.

Foerster R., Richtsteig E. (eds.) Libanii Opera omnia. Vol. IX. Leipzig, Teubner, 1927.

Gautier P. (éd.) Théophylacte d'Achrida. Lettres. Thessalonique, Association de Recherches Byzantines, 1986

Giannelli C. Codices Vaticani graeci. Codices 1684-1744. Typis Polyglottis Vaticanis 1961.

Grünbart M. Formen der Anrede im byzantinischen Brief vom 6. bis zum 12. Jahrhundert. Wien, Verlag der Österr. Akad. der Wiss., 2005.

Hunger H. Katalog der griechischen Handschriften der österreichischen Nationalbibliothek. Teil 1. Wien, Georg Prachner Verlag, 1961.

Hunger H. Die hochsprachliche profane Literatur der Byzantiner. Bd. 2. München, Beck, 1978.

Karlsson G. Idéologie et cérémonial dans l'épistolographie byzantine. Uppsala, Almqvist \& Wiksell, 1959.

Komines A.D. Gregorio Pardos, Metropolita di Corinto, e la sua opera. Roma, Institut di Studi Bizantini e Neoellenici, Univ. di Roma, 1960.

Kazhdan A. P. A history of Byzantine literature (850-1000). Athens, Institute for Byzantine Research, 2006. Koskenniemi, H. Studien zur Idee und Phraseologie des griechischen Briefes bis 400 n. Chr. Helsinki 1956. 


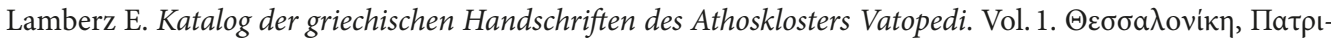

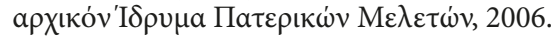

Lameere W. La tradition manuscrite de la correspondancen de Grégoire de Chypre, Patriarche de Constantinople (1283-1289). Bruxelles, Palais des Académies, 1937.

Laourdas B., Westerink L.G. Photii patriarchae Constantinopolitani epistulae et amphilochia. Vol.1-6. Leipzig, Teubner, 1983-1988.

Loenertz R.-J. Démétrius Cydonès. Correspondance. Città del Vaticano, Bibl. Apostolica Vaticana, 1956-1960.

Malherbe A. J. Ancient Epistolary Theorists. Atlanta, Scholars Press, 1988.

Mercati I., de Cavalieri P. F. Codices Vaticani graeci. Codices 1-329. Typis Polyglottis Vaticanis 1923.

Mioni E. Bibliothecae Divi Marci Venetiarum codices Graeci manuscripti. Vol.2. Roma, Istituto Poligrafico dello Stato, 1960.

Nares R. Catalogue of the Harleian Manuscripts in the British Museum. Vol. 1. London, J. Eyre and A. Strahen, 1808.

Oikonomidès N. Les listes de préséance Byzantines des IXe et Xe siècles. Paris, Centre National de la Recherche Scientifique, 1972.

Omont H. Inventaire sommaire des manuscrits grecs de la Bibliothèque Nationale. Vol.1-3. Paris, Picard, 1886-1888.

Rabe H. Aus Rhetoren-Handschriften: 9. Griechische Briefsteller. Rheinisches Museum für Philologie 1909, 64, 284-309.

Schartau B. Codices Graeci Haunienses. Ein deskriptiver Katalog des griechischen Handschriftenbestandes der Königlichen Bibliothek Kopenhagen. Copenhagen, Museum Tusculanum Press, 1994.

Schreiner P. Codices Vaticani Graeci. Codices 867-932. Typis Polyglottis Vaticanis, 1988.

Stevenson H. M. Codices manuscripti Palatini Graeci Bibliothecae Vaticanae descripti. Romae, Ex Typographeo Vaticano, 1885.

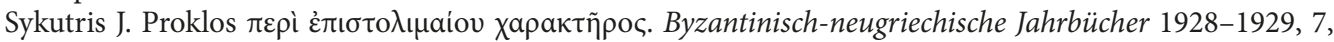
$108-118$.

Rostagno E., Festa N. Indice dei Codici greci Laurenziani non compresi nel Catalogo del Bandini. Studi italiani di Filologia classica 1893, 1, 129-232.

Thraede, K. Grundzüge griechisch-römischer Brieftopik. München, Beck, 1970.

Tziatzi-Papagianni M. (ed.). Theodori Metropolitae Cyzici epistulae. Berlin, de Gruyter, 2012.

Walz C. Rhetores Graeci, vol. 3. Stuttgart, Cotta, 1834.

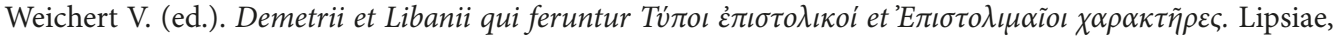
Teubner, 1910.

Zilliacus H. Untersuchungen zu den abstrakten Anredeformen und Höflichkeitstiteln im Griechischen. Helsingfors 1949.

For citation: Chernoglazov D.A. Die Byzantinische Fassung des spätantiken Briefstellers: Überlieferung und Textgeschichte. Philologia Classica 2017, 12(2), 188-205. https://doi.org/10.21638/11701/ spbu20.2017.208

\section{ВИЗАНТИЙСКАЯ РЕДАКЦИЯ ПОЗДНЕАНТИЧНОГО ПИСЬМОВНИКА: РУКОПИСНАЯ ТРАДИЦИЯ, ИСТОРИЯ ТЕКСТА}

Дмитрий Александрович Черноглазов

Санкт-Петербургский государственный университет, Российская Федерация, 199034, Санкт-Петербург, Университетская наб., 7-9; d_chernoglazov@mail.ru

«Эпистолярные стили» Псевдо-Либания (сокр. PL1) - трактат по эпистолярному искусству $\mathrm{V}$ в., включающий теоретическую часть и образцы писем. Образцы относятся к 41 типу, как правило, по одному образцу на каждый тип. Если сам трактат, составляющий основу античной эпистолярной теории, изучен хорошо, то его византийские и поствизантийские версии вовсе не исследованы. В настоящей статье анализируется одна из этих версий - сборник образцов писем, составленный на основе «Эпистолярных стилей» Псевдо-Либания (сокр. PL2). Исследование включает следующие аспекты: 1) Содержание и стиль PL2. Наименования типов, представленных в PL2, и тексты образцов восходят в основном к трактату Псевдо-Либания. Анализируется метод переработки источника: текст расширяется риторическими средствами - регулярно вводятся эпитеты, сравнения, пословицы и цитаты. 2) Рукописная традищия PL2. B рукописях PL2, как правило, присоединяется к трактату Псевдо-Либания и вступает с ним в различные комбинации. В зависимости от струк- 
туры текста, списки PL2 подразделяются на 5 классов. Дается характеристика каждой рукописи: учитывается не только сам письмовник, но и его сопровождение в рукописях. 3) История текста PL2. Делается попытка реконструкции архетипа PL2: демонстрируется, что изначально это было не приложение к «Эпистолярным стилям», а самостоятельный письмовник; предполагаемое время его создания - Х в. 4) Назначение и функции PL2. Предполагается, что PL2 создавался с целью актуализировать античный письмовник. В рукописях PL2 нередко выступает как переходное звено между простейшими образцами Псевдо-Либания и подлинными письмами византийских авторов. Возможно, трактат использовался также и как школьный учебник. В Приложении перечисляются все образцы писем, входящие в PL2, с их названиями и incipit. B дальнейшем автор статьи планирует подготовить критическое издание как «Эпистолярных стилей», так и PL2 и других византийских версий трактата.

Ключевые слова: эпистолярная теория, письмовник, рукописная традиция, текстология, античная эпистолография, византийская эпистолография, Псевдо-Либаний, Characteres epistolici.

Received: 12.06 .2017

Final version received: 08.09.2017 